MODELING, IDENTIFICATION AND CONTROL, 1995, VOL. 16, NO. 2, 107-117

doi:10.4173/mic.1995.2.3

\title{
The control system of an autonomous underwater vehicle
}

\author{
BJØRN JALVING† and NILS STØRKERSEN†
}

Keywords: Autonomous underwater vehicle, flight control system, sea trials

This paper presents the flight control system of an Autonomous Underwater Vehicle (AUV) developed at the Norwegian Defence Research Establishment. A mathematical model of the vehicle is derived and discussed. The system is separated into lightly interacting subsystems and three autopilots are designed for steering, diving and speed control. The design of the separate controllers is based on PID techniques. Results from sea trials show robust performance and stability for the autopilot.

\section{Introduction}

In recent years the interest in Autonomous Underwater Vehicles (AUV) has grown as new technology has made practical solutions more feasible. AUVs are untethered free swimmers with sufficient on-board intelligence to execute complex missions. Several military and civilian applications have been identified. Among these are search and reconnaissance, mine field survey, bottom mapping, package delivery/retrieval, better and cheaper oceanographic data gathering and pipeline inspection.

In January 1992 the Norwegian Defence Research Establishment (NDRE) began developing an AUV. The main target of the programme was to demonstrate a propulsion system based on a new sea water battery technology. The sea water battery was initially developed for use in low power, long term stationary applications, for instance in light buoys, oceanographic instrumentation, oil installations and underwater surveillance systems, but in this programme was adapted for use in AUVs (Hasvold 1993).

The main subject of this paper is the design and implementation of a control system for autonomous operation. Figure 1 shows the NDRE-AUV being deployed. The AUV has a low drag hull in order to minimize power losses. The vehicle has a length of hull of $4.315 \mathrm{~m}$, a maximum hull diameter of $0.735 \mathrm{~m}$, a nominal cruise speed of approximately 4 knots $(2 \cdot 1 \mathrm{~m} / \mathrm{s})$ and a hull contour displacement of $1.021 \mathrm{~m}^{3}$.

\section{NDRE-AUV system overview}

The high level control system in the NDRE-AUV has a sufficient degree of autonomy to test the potential capabilities of the sea water battery for long range missions. In the next generation more effort will be put into the design of expert and navigation systems. Figure 2 shows a functional diagram of the high level control system for the NDRE-AUV.

The mission begins with programming the Mission Executor, followed by the vehicle launch. The Mission Executor is programmed by a TEXAS laptop MS-DOS

Received 15 November 1994.

† Norwegian Defence Research Establishment, P. O. Box 25, N-2007 Kjeller, Norway, Bjorn.Jalving@ffi.no, Nils-J.Storkersen@ffi.no.

(C) 1994 IEEE. Reprinted, with permission, from Proceedings of the Third IEEE Conference on Control Applications, Glasgow, 24-26 August 1994, pp. 851-856. 


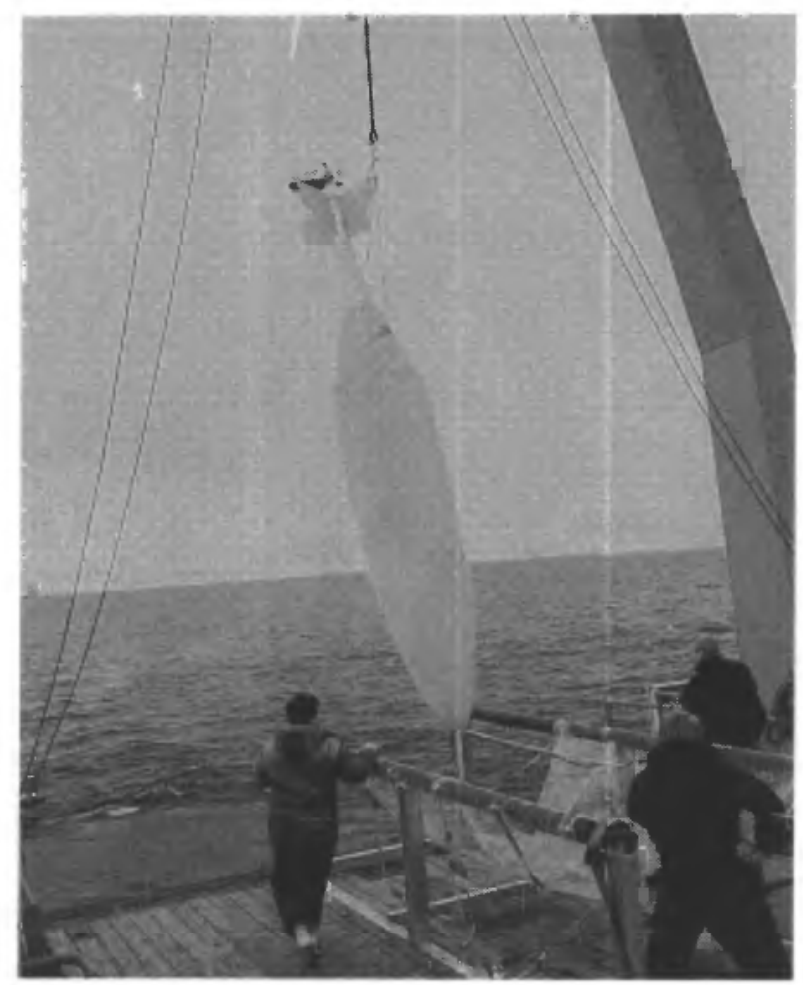

Figure 1. The picture shows the deployment of the NDRE-AUV in the open sea between Norway and Denmark.

machine through the Serial Link Interface. The single on-board computer is based on the Intel i80c196 embedded controller. Figure 2 shows that the Serial Link Interface is also used to configure the Data Storage system and the Fault Detection system. 2 MB of flash-memory is used to store sensor data. the Fault Detection system monitors the most important system parameters. In the event of a serious error it brings the vehicle to the surface by shutting down propulsion and releasing a drop weight.

The parameters of the Vehicle Control System are also programmable. In the figure, the term Vehicle Control System comprises control of a DC/DC converter for the sea water battery as well as the autopilot. The major interfacing in the execution phase is between the Sensors, the Vehicle Control System, the Mission Executor and the control inputs.

The AUV navigates by following a specified course at a specified depth for a period of time. The sensors used for navigation and flight control are a gimballed flux-gate compass, a depth sensor, an inclinometer and two rate sensors. The measurements are described in more detail in $\$ 5$. The vehicle's navigation system sufficed for testing the propulsion system but advanced missions require a more sophisticated solution. We have been working with aided inertial navigation systems and techniques for terrain matching (Bergem 1993).

A Hydro-acoustic Position Reference (HPR) system, manufactured by Simrad Subsea A/S, is also incorporated. This system has two main functions: to keep track of the AUV from the mother vessel and to make it possible to interfere with the Mission Executor by commanding the AUV. 


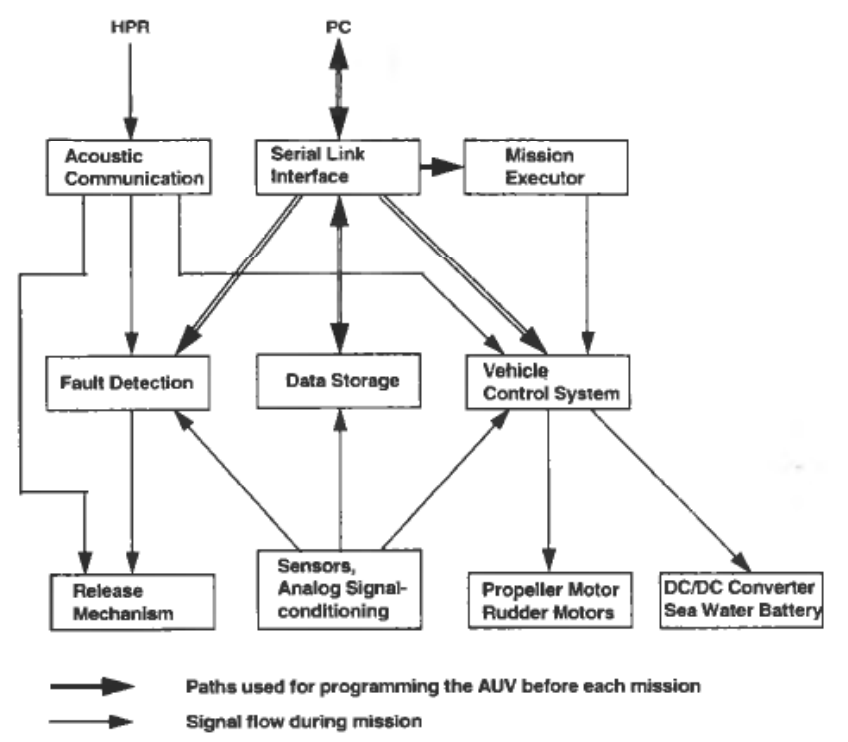

Figure 2. Functional diagram of the NDRE-AUV high level control system.

\section{Flight control philosophy}

The design of an autopilot for the motion control of an underwater vehicle must consider both motion stabilisation and manoeuvring. The controller had to be made robust enough to deal with significant modeling uncertainty.

The 6 degrees of freedom differential equations of motion are divided into three non-interacting or lightly interacting subsystems for speed control, steering and diving. This approach leads to a set of separate designs for steering, diving and speed control systems. This control philosophy accords with the current practice in naval submarines.

Each subsystem is treated as a single input multi-state (SIMS) system. The design of the separate controllers is based on PID techniques.

\section{Vehicle modeling}

The notation used in this paper is in accordance with SNAME (1950). The 6 degrees of freedom (DOF) non-linear equations of motion are most conveniently developed using a body-fixed coordinate frame and a global reference frame. The body-fixed frame has components of motion given by the 6 velocity components $[u, v, w, p, q, r]$ (surge, sway, heave, roll, pitch and yaw), relative to a constant velocity coordinate frame moving with the ocean current, $\mathbf{u}_{c}$. The velocity vector is represented as

$$
v=[u, v, w, p, q, r]^{\mathrm{T}}
$$

while the six components of position and attitude in the global reference frame are

$$
\eta=[x, y, z, \phi, \theta, \psi]^{\mathrm{T}}
$$

The vectors are related through the Euler angle transformation,

$$
\dot{\boldsymbol{\eta}}=\boldsymbol{J}(\boldsymbol{\eta}) \boldsymbol{v}
$$


Control effectors such as control surface, propeller speeds, thruster forces and buoyancy adjustment are usually considered as the vector $\boldsymbol{u}$. For the NDRE-AUV, $\boldsymbol{u}$ is given by:

$$
\boldsymbol{u}=\left[\delta_{r}(t), \delta_{s}(t), n(t)\right]^{\mathrm{T}}
$$

where $\delta_{r}(t)$ is the rudder deflection, $\delta_{s}(t)$ is the stern plane deflection and $n(t)$ is the propeller revolution. The vehicle is approximately neutrally buoyant. Otherwise, stern plane deflection would have increased the drag and thus the power consumption significantly.

The non-linear vehicle dynamics can be expressed in a compact form as (Fossen 1991).

$$
M \dot{v}+C(v) v+D(v) v+g(v)=B(v) \mathbf{u}
$$

where $M$ is a $6 \times 6$ inertia matrix including hydrodynamic added mass, $C(v)$ is a matrix of the Coriolis and centripetal forces, $D(v)$ is the hydrodynamic damping matrix, $g(\eta)$ is a vector of restoring forces and moments and $\boldsymbol{B}(\eta)$ is a $6 \times 3$ control matrix. For details on the modeling aspects, the interested reader is recommended to consult Fossen (1994).

Equation (5) is not practical for conventional decoupled control system design. We look for an alternative solution by separating the system into non-interacting (or lightly interacting) subsystems, grouping certain key motion equations together for the separate functions of steering, diving and speed control. This decomposition is motivated by the slender form of our vehicle, but has also been reported elsewhere (Rodriguez and Dobeck (1989), Healy and Marco (1992), Healy and Lienard (1993). The three subsystems and their state variables are given by

(1) Speed system state: $u(t)$

(2) Steering system states: $v(t), r(t)$ and $\psi(t)$

(3) Diving system states: $w(t), q(t), \theta(t)$ and $z(t)$

The roll dynamic, $\phi(t)$, is neglected. This is justified by the designing the vertical distance between the centre of gravity and the centre of buoyancy, denoted $\overline{B G}_{z}$ sufficiently long to suppress the moment from the propeller. For a neutrally buoyant vehicle it can be shown that the relationship between $\overline{B G}_{z}$ and the steady-state roll angle is given by

$$
\phi_{0}=\arcsin \left(P / W \overline{B G}_{z} \omega_{p}\right)
$$

where $P$ is propeller power, $W=m g$ is the gravitational force and $\omega_{p}$ is the propeller revolution in radians per second.

\section{Flight control systems}

One controller was designed for each of the three subsystems. The steering autopilot is responsible for the control of heading errors, the diving system is responsible for depth errors and the speed system controls a propeller DC motor with graphite brushes. There were assumed to be no interactions between the controller, see the discussion on results in $\S 6$.

Robustness was ensured by applying more sensors than strictly necessary and designing high margins of stability. 


\subsection{Speed control autopilot}

Not taking into account the interactions from the other degrees of freedom (DOF), the longitudinal rigid-body equation of motion is given by

$$
m \dot{u}=X
$$

where $X$ represents external surge forces acting on the vehicle and $m$ is the mass of the vehicle. Including both linear and quadratic damping $\left(X_{u}\right.$ and $\left.X_{|u| u}\right), X$ can be described as

$$
X=X_{u} \dot{u}+X_{u} u+X_{|u| u \mid}|u| u+T_{|n| n}|n| n+T_{u n \mid}|n| u
$$

where $X_{u}$ is hydrodynamic added mass and $T_{|n| n}$ and $T_{u n}$ are two propeller coefficients.

From eqns (7) and (8) we observe that there is a relationship between the propeller revolution and the surge speed $u$. The vehicle has a DC motor controller, but there is no explicit control of $u$. However, in order to optimize the sea water battery operation, a power control loop with the desired propeller revolution $n_{d}$ as a control variable was designed.

The transfer function of a DC motor is given by (Egeland 1993).

$$
h_{m}(s)=\frac{n(s)}{u_{a}(s)}=\frac{K}{\left(1+T_{m} s\right)\left(1+T_{a} s\right)}
$$

where $K$ is a motor constant, $T_{m}$ is the mechanical time constant and $T_{a}$ is the electrical time constant. Usually $T_{a}<0 \cdot 1 \cdot T_{m}$, thus $T_{a}$ is often disregarded in the control design. $u_{a}(s)$ is the input voltage, in practice controlled by pulse-width modulation (PWM).

A PI-controller with time constant $T_{i}$ and gain constant $K_{p}$,

$$
h_{c}(s)=\frac{u_{a}(s)}{n_{d}(s)-n(s)}=\frac{K_{p}\left(1+T_{i} s\right)}{T_{i} s}
$$

was applied. Proper tuning of the PI-control parameters ensured that $n(t)$ tracked the desired propeller revolution $n_{d}(t)$, see Figure 3 .

\subsection{Steering autopilot}

The rigid-body ship dynamics in sway and yaw can be simplified to (Fossen 1994)

$$
\begin{aligned}
m \dot{v}+m u_{0} r & =Y \\
I_{z} \dot{r} & =N
\end{aligned}
$$

where $u_{0}$ is the constant surge speed. For small roll and pitch angles, we have that

$$
\psi=\frac{\sin \phi}{\cos \theta} q+\frac{\cos \phi}{\cos \theta} r \approx r
$$

Linear modeling of hydrodynamic added mass, damping and the rudder yields

$$
\begin{aligned}
& Y=Y_{\dot{v}} \dot{v}+Y_{\dot{r}} \dot{r}+Y_{v} v+Y_{r} r+Y_{\delta} \delta_{r} \\
& N=N_{\dot{v}} \dot{v}+N_{\dot{r}} \dot{r}+N_{v} v+N_{r} r+N_{\delta} \delta_{r}
\end{aligned}
$$

Quadratic damping coefficients can be disregarded because of limited magnitude of 
$v$ and $r$. Equations (11), (12) and (13) can be expressed in a compact form as

$$
\left[\begin{array}{ccc}
m-Y_{\dot{v}} & -Y_{\dot{r}} & 0 \\
-N_{\dot{v}} & I_{z}-N_{\dot{r}} & 0 \\
0 & 0 & 1
\end{array}\right]\left[\begin{array}{c}
\dot{v} \\
\dot{r} \\
\psi
\end{array}\right]+\left[\begin{array}{ccc}
-Y_{v} & -Y_{r}+m u_{0} & 0 \\
-N_{v} & -N_{r} & 0 \\
0 & -1 & 0
\end{array}\right]\left[\begin{array}{c}
v \\
r \\
\psi
\end{array}\right]=\left[\begin{array}{c}
Y_{\delta} \\
N_{\delta} \\
0
\end{array}\right] \delta_{r}
$$

Rearranging this expression in state space form

$$
\dot{\mathbf{x}}=\mathbf{A x}+\mathbf{b} \delta_{r}
$$

where $\mathbf{x}=[v, r, \psi]^{\mathrm{T}}$ yields

$$
\left[\begin{array}{c}
\dot{v} \\
\dot{r} \\
\psi
\end{array}\right]=\left[\begin{array}{ccc}
a_{11} & a_{12} & 0 \\
a_{21} & a_{22} & 0 \\
0 & 1 & 0
\end{array}\right]\left[\begin{array}{c}
v \\
r \\
\psi
\end{array}\right]+\left[\begin{array}{c}
b_{1} \\
b_{2} \\
0
\end{array}\right] \delta_{r}
$$

where the definitions of $a_{i j}$ and $b_{i}$ should be quite obvious.

The heading $\psi$ was measured with a gimballed flux-gate compass. In addition the yaw rate $r$ was measured with a rate sensor. The heading measurement would have been sufficient for designing a controller, but adding a rate measurement to the controller, increases robustness. The control law was simply taken to be

$$
\delta_{r}=K_{p}\left(\psi_{d}-\psi\right)-K_{d} r
$$

where $K_{p}$ and $K_{d}$ are the proportional and derivative gain, respectively.

The control law has no integral action. Hence, steady state errors due to environmental disturbances and neglected dynamics cannot be compensated for. The main reason for omitting integral action is that the rudder servo has an on-off or relay nonlinearity which would cause limit cycles (chattering) if integral action was added. In addition to this, the magnitude of the steady-state errors, 1-2 degrees, was of the same order of magnitude as the accuracy of the flux-gate compass. Consequently, there is not much gained by including integral action.

The loop transfer function is given by

$$
\begin{aligned}
c(s) & =\frac{\psi}{\psi_{d}-\psi}(s) \\
& =\frac{K_{p}\left(b_{2} s-a_{11} b_{2}+a_{21} b_{1}\right)}{s^{3}+\left(K_{d} b_{2}-a_{11}-a_{22}\right) s^{2}+\left(a_{11} a_{22}-a_{12} a_{21}+K_{d} a_{21} b_{1}-K_{d} a_{11} b_{2}\right) s}
\end{aligned}
$$

The controller parameters were chosen by using standard techniques such as pole-placement and AFF-diagrams.

In order to suppress the effects of high-frequency disturbances (induced action from the waves when the vehicle is close to the surface) and discretization steps, the measurements were digitally lowpass filtered in addition to the analogue lowpass filtering which was included to prevent aliasing. The desired heading, $\psi_{d}$ was also lowpass filtered in order to obtain smooth course-changing manoeuvres.

\subsection{Diving autopilot}

The simplified rigid-body equations of motion in heave and pitch is given by Fossen (1994)

$$
\begin{aligned}
m\left(\dot{w}-u_{0} q\right) & =Z \\
I_{y} \dot{q} & =M
\end{aligned}
$$


As with the steering model, the external forces and moments are described by hydrodynamic added mass, linear damping and the effects of the stern plane deflection. In addition, the moment caused by the vertical distance between the centre of gravity and the centre of buoyancy, $\overline{B G}_{z}=z_{G}-z_{B}$, must be modeled.

$$
\begin{aligned}
Z & =Z_{\dot{w}} \dot{w}+Z_{\dot{q}} \dot{q}+Z_{w} w+Z_{q} q+Z_{\delta} \delta_{s} \\
M & =M_{\dot{w}} \dot{w}+\dot{M}_{\dot{q}} \dot{q}+M_{w} w+M_{q} q-m g\left(z_{G}-z_{B}\right) \sin \theta+M_{\delta} \delta_{s} \\
& \approx M_{\dot{w}} \dot{w}+\dot{M}_{\dot{q}} \dot{q}+M_{w} w+M_{q} q-W \overline{B G}_{z} \theta+M_{\delta} \delta_{s}
\end{aligned}
$$

In steady state we have $\theta_{0}=q_{0}=\phi_{0}=0$. This suggests the following relations (dropping 4 -notation):

$$
\begin{aligned}
& \dot{\theta}=q \\
& \dot{z}=-\theta u_{0}+w
\end{aligned}
$$

Equations (19), (20) and (21) can be written as:

$$
\left[\begin{array}{cccc}
m-Z_{\dot{w}} & -Z_{\dot{q}} & 0 & 0 \\
-M_{\dot{w}} & I_{y}-M_{\dot{q}} & 0 & 0 \\
0 & 0 & 1 & 0 \\
0 & 0 & 0 & 1
\end{array}\right]\left[\begin{array}{c}
\dot{w} \\
\dot{q} \\
\dot{\theta} \\
\dot{z}
\end{array}\right]+\left[\begin{array}{cccc}
-Z_{w} & m u_{0}-Z_{q} & 0 & 0 \\
-M_{w} & -M_{q} & \overline{B G}_{z} W & 0 \\
0 & -1 & 0 & 0 \\
-1 & 0 & u_{0} & 0
\end{array}\right]\left[\begin{array}{c}
w \\
q \\
\theta \\
z
\end{array}\right]=\left[\begin{array}{c}
Z_{\delta} \\
M_{\delta} \\
0 \\
0
\end{array}\right] \delta_{s}
$$

This implies a state space model

$$
\left[\begin{array}{c}
\dot{w} \\
\dot{q} \\
\dot{\theta} \\
\dot{z}
\end{array}\right]=\left[\begin{array}{cccc}
c_{11} & c_{12} & c_{13} & 0 \\
c_{21} & c_{22} & c_{23} & 0 \\
0 & 1 & 0 & 0 \\
1 & 0 & -u_{0} & 0
\end{array}\right]\left[\begin{array}{l}
w \\
q \\
\theta \\
z
\end{array}\right]+\left[\begin{array}{l}
d_{1} \\
d_{2} \\
0 \\
0
\end{array}\right] \delta_{s}
$$

with obvious definitions of $c_{i j}$ and $d_{i}$. Both simulations and sea trials indicated that the heave velocity, $w$, during diving is small (less than $0.05 \mathrm{~m} / \mathrm{s}$ ). This implies that the linear model in (23) reduces to

$$
\left[\begin{array}{c}
\dot{q} \\
\dot{\theta} \\
\dot{z}
\end{array}\right]=\left[\begin{array}{ccc}
\frac{M_{q}}{I_{y}-M_{\dot{q}}} & -\frac{\overline{B G_{z}} W}{I_{y}-M_{\dot{q}}} & 0 \\
1 & 0 & 0 \\
0 & -u_{0} & 0
\end{array}\right]\left[\begin{array}{l}
q \\
\theta \\
z
\end{array}\right]+\left[\begin{array}{c}
\frac{M_{\delta}}{I_{y}-M_{\dot{q}}} \\
0 \\
0
\end{array}\right] \delta_{s}
$$

This model is well suited for control design. The transfer functions $\theta(s) / \delta_{s}(s)$ and $z(s) / \delta_{s}(s)$ are obtained as follows:

$$
\frac{\theta(s)}{\delta_{s}(s)}=\frac{K_{\theta}}{s^{2}+2 \varsigma_{\theta} \omega_{\theta} s+\omega_{\theta}^{2}} ; \quad \frac{z(s)}{\delta_{s}(s)}=-\frac{u_{0}}{s} \frac{\theta(s)}{\delta_{s}(s)}
$$

where the gain constant is: $K_{\theta}=M_{\delta} /\left(I_{y}-M_{\dot{q}}\right)$ and the natural frequency $\omega_{\theta}$ and relative damping ratio $\varsigma_{\theta}$ are defined as

$$
\omega_{\theta}=\left(\frac{\overline{B G}_{z} W}{I_{y}-M_{q}}\right)^{1 / 2} ; \quad \varsigma_{\theta}=\frac{-M_{q}}{2\left(\overline{B G}_{z} W\left(I_{y}-M_{\dot{q}}\right)\right)^{1 / 2}}
$$


Three measurements were used in the depth controller. The depth $z$ was measured by a pressure meter, the pitch angle $\theta$ by an inclinometer and the pitch rate $q$ by a rate sensor. The depth controller was designed without integral action according to:

$$
\delta_{s}=G_{1}\left(z_{d}-z\right)-G_{2} \theta-G_{3} q
$$

Inserting this expression into (25) yields

$$
\left[s^{2}+\left(2 \varsigma_{\theta} \omega_{\theta}+K_{\theta} G_{3}\right) s+\left(\omega_{\theta}^{2}+K_{\theta} G_{2}\right)\right] \theta(s)=K_{\theta} G_{1}\left[z_{d}(s)-z(s)\right]
$$

Hence, we can choose $G_{2}$ and $G_{3}$ so that the closed loop pitch dynamics is stable. Next, we can use the relationship:

$$
z(s)=-\frac{u_{0}}{s} \theta(s)
$$

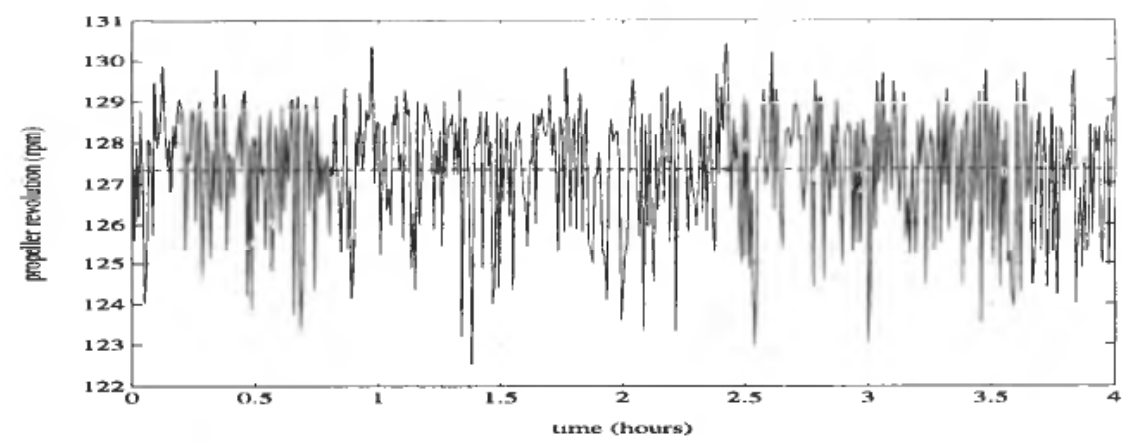

Figure 3. Desired and actual propeller revolution versus time.
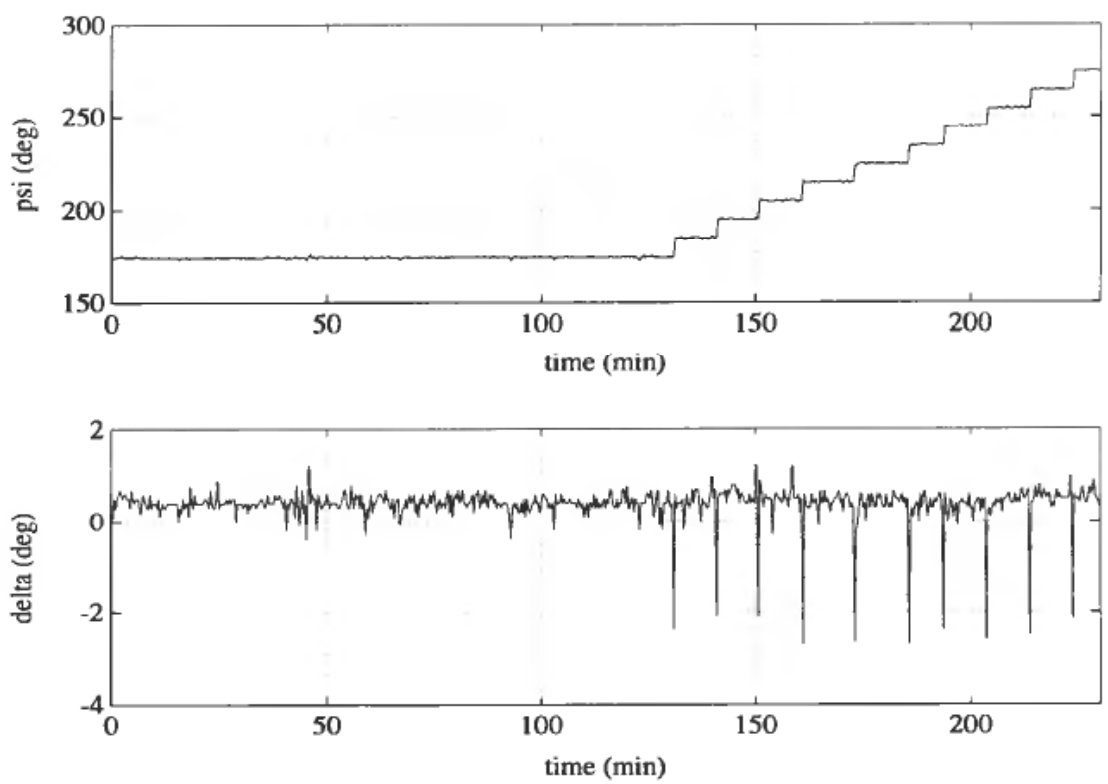

Figure 4. Part of a long time mission where the heading is changed in steps of $10^{\circ}$. 
to tune $G_{1}$. Moreover, the cubic characteristic equation

$$
\left[s^{3}+\left(2 \varsigma_{\theta} \omega_{\theta}+K_{\theta} G_{3}\right) s^{2}+\left(\omega_{\theta}^{2}+K_{\theta} G_{2}\right) s-u_{0} K_{\theta} G_{1}\right] z(s)=-u_{0} K_{\theta} G_{1} z_{d}(s)
$$

must be stable to ensure that $\left(z=z_{d}=\right.$ const) in steady state. However, environmental disturbances can cause steady state errors for this approach since integral action is omitted. This is also the case if the inclinometer or the rate sensor has a bias. The main reason for omitting integral action is that the stern plane servo has a relay nonlinearity, which would cause limit cycles if integral action was added.

\section{Results from sea trials}

The NDRE-AUV was tested in a total of 26 missions of varying duration in April and May 1993. The flight control system performed successfully on the first attempt due to extensive use of simulators for testing on shore. The rest of the systems in the AUV also behaved satisfactorily.

During two and a half days the NDRE-AUV travelled a distance of 109 nautical miles in three consecutive missions in the open sea between Norway and Denmark. At this point the depth of discharge of the battery was only approximately $10 \%$ of the nominal capacity, indicating a much longer potential vehicle range, with this new sea water battery system.

The assumption of light interaction between the autopilots was verified. This was accomplished because the roll angle was always less than $2^{\circ}$ (confirming eqn (6)), while the pitch angle during descent and ascent never exceeded $20^{\circ}$.

The performance of the speed controller for a typical mission is shown in Fig. 3, where the referenced propeller revolution is $n_{d}=127.3 \mathrm{rpm}$. Improvements in the control deviation can be achieved by increasing the bandwidth of the controller, but this requires a higher sample-rate.

The performance of the heading control system is shown in Fig. 4 for a mission where the course is changed in steps of $10^{\circ}$ approximately every $10 \mathrm{~min}$. A typical step response is shown in Fig. 5, where the heading angle is changed from $195^{\circ}$ to $185^{\circ}$. We observe that the course changing takes approximately $10 \mathrm{sec}$. More rapid control can be achieved, but robustness was given main priority in the autopilot design.

A full-scale depth changing manoeuvre where the NDRE-AUV dives to $50 \mathrm{~m}$ is shown in Fig. 6. The figure shows the dynamic behaviour of the state variables in eqn. (24) and the stern plane activity.

There was calm sea state during the trials presented in the figures. However, the AUV was tested with a significant wave height of approximately $1.5 \mathrm{~m}$. Even though the rudder and sternplane activity became more oscillatory, the flight control system behaved satisfactorily near the surface.

\section{Conclusion}

A flight control system for an AUV has been designed and implemented. The system proved robust and had stable performance during extensive open sea testing.

The NDRE-AUV met its objectives by travelling a distance of more than 100 nautical miles, thus demonstrating the capability of the novel sea water battery technology. The capacity of the sea water battery, however, provides a much longer vehicle range. 

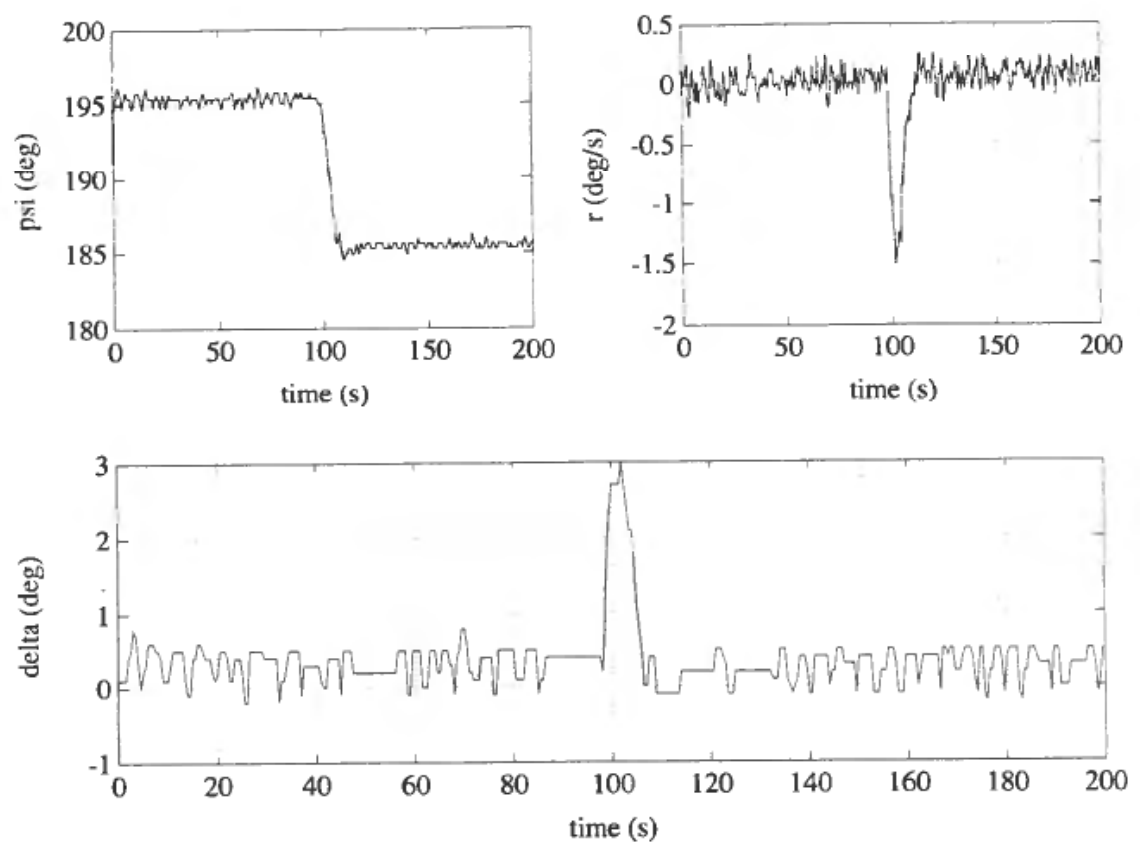

Figure 5. One typical step response where the heading is changed by $10^{\circ}$.
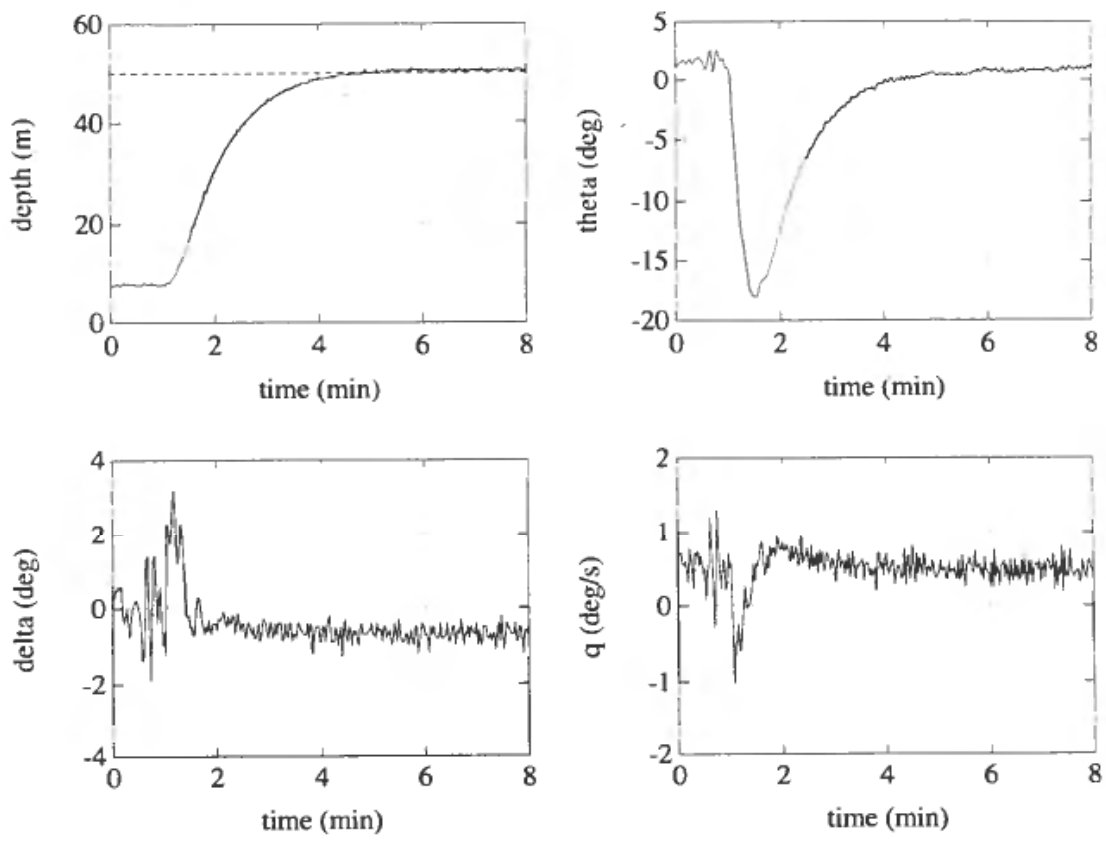

Figure 6. Full-scale depth changing manoeuvre for the NDRE-AUV. The bias in the pitch rate time series is due to a small off-set in the rate sensor. 


\section{Acknowledgement}

The authors wish to thank Dr T. I. Fossen at the Norwegian Institute of Technology for his helpful comments.

\section{REFERENCES}

Bergem, O. (1993), A multibeam sonar based positioning system for an AUV, in Eighth International Symposium on Unmanned Untethered Submersible Technology, Autonomous Undersea Systems Institute, Portsmouth, New Hampshire, pp. 291-299, Sep. 1993.

Egeland, O. (1994), Servoteknikk (in Norwegian) (Tapir, Trondheim).

Fossen, T. I. (1994), Guidance and Control of Ocean Vehicles (John Wiley \& Sons Ltd., London).

Fossen, T. I. (1991), Nonlinear modelling and control of underwater vehicles, Ph.D. Thesis, The Norwegian Institute of Technology, Dept. of Engineering Cybernetics, Trondheim.

HASVOLD, Ø. (1993). A magnesium-seawater power source for autonomous underwater vehicles, in The 18th International Power Sources Symposium, Stratford-upon-Avon, pp. 243-255, April 1993.

HeAley, A. J. and MaRCo, D. B. (1992), Slow speed flight control of autonomous underwater vehicles: experimental results with NPS AUV II, in Proceedings of the Second International Offshore and Polar Engineering Conference, San Francisco, June 14-19 1992, pp. 523-532.

HEALY, A. J. and LIENARD, D. (1993), Multivariable sliding mode control for autonomous diving and steering of unmanned underwater vehicles, IEEE Journal of Oceanic Engineering, 18, 327-339.

RODRIGUEZ, R. R. and DOBEcK, G. J. (1989), Guidance and control system of the large scale vehicle, in Sixth International Symposium on Unmanned Untethered Submersible Technology, Marine Systems Engineering Laboratory, University of New Hampshire, June 1989. pp. $434-451$.

SNAME, 1950, The Society of Naval Architects and Marine Engineers, Nomenclature for treating the motion of a submerged body through a fluid, Technical and Research Bulletin, 1-5. 